Lynn, V. L., M. D. Sohigian, and E. A. Crocker (1963), Radar observations of the moon at $8.6 \mathrm{~mm}$ wavelength, MIT Lincoln Lab. Tech. Rept. 331, ASTIA No. DDC 426207 (1963). See also, J. Geophys. Res. 69, 781-783 (1964).

Markov, A. V. (1948), Brightness distribution over the lunar disk at full moon, Astron. Zh. (USSR) 25, 172-179.

Muhleman, D. O. (1964), Radar scattering from Venus and the moon, Astron. J. 69, 34-41.

Norton, K. A. and A. C. Omberg (1947), The maximum radar set, Proc. IRE 35, 4-24.

Pettengill, G. H. (1960), Measurements of lunar reflectivity using the Millstone radar, Proc. IRE 48, 933-934.

Pettengill, G. H., and J. C. Henry (1962a), Radio measurements of the lunar surface, The Moon, IAU Symposium 14, ed. Z. Kopal and Z. K. Mikailov, 519-525 (Academic Press, London).

Pettengill, G. H., and J. C. Henry (1962b), Enhancement of radar reflectivity associated with the lunar crater Tycho, J. Geophys. Res. 67, 4881-4885.

Pettit, E. (1961), Planetary temperature measurements, The Solar System, vol. 3, Planets and Satellites, ed. G. P. Kuiper and B. M. Middlehurst, ch. 10 (Univ. of Chicago Press, Chicago).

Rea, D. G., N. Hetherington, and R. Mifflin (1964), The analysis of radar echoes from the moon, J. Geophys. Res. 69, 5217-5223.

Salomonovich, A. E., and B. Y. Losovsky (1962), Radio brightness distribution of the lunar disk at $0.8 \mathrm{~cm}$, Astron. Zh. (USSR) 39, 1074-1082.

Senior, T. B. A., and K. M. Siegel (1959), Radar reflection characteristics of the moon, Paris Symposium on Radio Astronomy, ed. R. N. Bracewell, p. 29 (Stanford Univ. Press, Stanford, Calif.).

Senior, T. B. A., and K. M. Siegel (1960), A theory of radar scattering by the moon, J. Res. NBS 64D (Radio Prop.), No. 3, 217-228.
Shorthill, R. W. (1962), Measurements of lunar temperature variations during an eclipse and throughout a lunation, Boeing Report D.1. $82-0196$.

Soboleva, N. S. (1962), Measurement of the polarization of lunar radio emission on a wavelength of $3.2 \mathrm{~cm}$, Astron. Zh. (USSR) 39, 1124-1126.

Trexler, J. H. (1958), Lunar radio echoes, Proc. IRE 46, 286-292.

Troitsky, V. S. (1962), Radio emission of the moon, its physical state and the nature of its surface, The Moon, IAU Symposium 14, ed. Z. Kopal and Z. K. Mikhailov, p. 475 (Academic Press, London).

Twersky, V. (1962), On scattering of waves by random distributions, II. Two space scatterer formalism, J. Math. Phys. 3, 724-734.

Victor, W. K., R. Stevens, and S. W. Golomb (1961), Radar exploration of Venus, Jet Propulsion Lab Tech. Rept. 32-132.

Winter, D. F. (1962), A theory of radar reflections from a rough moon, J. Res. NBS 66D (Radio Prop.), No. 3, 215-226.

Yaplee, B. S., R. H. Bruton, K. J. Craig, and N. G. Roman (1958), Radar echoes from the moon at a wavelength of $10 \mathrm{~cm}$, Proc. IRE 46, 293-297.

Yaplee, B. S., N. G. Roman, K. J. Craig, and T. F. Scanlon (1959), A lunar radar study at $10 \mathrm{~cm}$ wavelength, Paris Symposium on Radio Astronomy, ed. R. N. Bracewell, p. 19 (Stanford Univ. Press, Stanford, Calif.).

Yaplee, B. S., S. H. Knowles, A. Shapiro, K. J. Craig, and D. Brouwer (1964), The mean distance to the moon as determined by radar, Naval Research Lab Rept. 6134.

\title{
Decameter-Wave Radar Studies of the Lunar Surface
}

\author{
J. R. Davis, D. C. Rohlfs, G. A. Skaggs, and J. W. Joss ${ }^{1}$
}

Radar Division, U.S. Naval Research Laboratory, Washington, D.C.

\begin{abstract}
An extended series of decameter-wave measurements of the total radar cross section of the Moon has corroborated a previous suggestion that this parameter has a substantially larger value in the decameter region than at shorter wavelengths. Examples are given of the ionospheric effects which require decameter-wave measurements conducted over a transionospheric path to be regarded with caution. A beginning study of possible discrete scattering centers located in regions toward the limb of the Moon is described.
\end{abstract}

\section{Introduction}

The U.S. Naval Research Laboratory, as part of a continuing program of research in radio propagation and radar techniques, has conducted a high-frequency (HF) radar study of the Moon and transionospheric propagation for the past $3 \frac{1 / 2}{2}$ years. Measurements of Moon-reflected radar signals at wavelengths between 11 and $22 \mathrm{~m}$ have been made on a regular basis during this period with the use of NRL's high-power HF transmitting facility. As is often the case with decameter-wave radio undertakings, however, the pattern of the large broadside array which has been used for this series of measurements has imposed severe restrictions on the operating schedule. Observations have been possible only for periods of approximately $1 \frac{1}{2} \mathrm{hr}$ immediately after moonrise on the few days each month when the Moon's delination exceeds $15^{\circ}$ North. Due to the ionospheric phenomena which affect decameter-wave radio signals, useful observations have been possible only during periods well after ionospheric sunset, as well.

${ }^{1}$ RCA Service Company.
In brief, the NRL high-power HF transmitting facility has been utilized in a monostatic, pulsed mode for this study, providing pulses of approximately $250 \mu \mathrm{sec}$ in length at pulse repetition frequencies of $5 \% / 8$ pulses per second and harmonically related multiples of that rate. Observations have centered largely upon measurements of two principal characteristics of the Moon as a decameter-wave radar target:

(a) Its total radar cross section, and

(b) its properties as a distributed scatterer.

An earlier series of measurements conducted with this facility [Davis and Rohlfs, 1964] has suggested that both of these parameters display somewhat different character at decameter wavelengths than in the meter and centimeter regions. In particular, the Moon's total radar cross section has been measured to be substantially larger at decameter wavelengths than at shorter wavelengths, and the relative contribution to the total scattered energy by areas beyond the Moon's central, specularly reflecting region has been measured to be distinctly less in the decameter region than at shorter wavelengths. This behavior has led to the suggestion than the Moon, apparently a perfectly rough or uniformly bright reflector at optical wave- 
lengths, displays a central highlight which brightens and diminishes in area with increasing wavelength at radio frequencies.

It is particularly with regard to the former résult, namely that the Moon's total radar cross section is larger in the decameter region than at shorter wavelengths, that these measurements have represented a matter for further concern to lunar radar workers. Most measurements in other bands, from millimeter through meter wavelengths, have shown no discernible wavelength-dependence in the Moon's total radar cross section. It has been to the task of clarifying this apparent disagreement that much of the recent decameter-wave lunar radar work at NRL has been addressed, and a recent series of measurements is discussed in some detail in section 2 .

Modifications to the HF radar facility also have permitted a refinement in NRL's studies of the Moon's behavior as a scatterer in depth, and work has begun on investigating possible discrete scattering centers or in-phase groups of scatterers which appear at ranges well beyond the Moon's central, specularly reflecting region. Initial results of these studies appear in section 3. Throughout the following discussion, stress is laid on the overriding concern of a decameter-wave radar astronomer: the precautions which are necessary to avoid contamination of his data by unrecognized perturbations in the terrestrial ionosphere.

\section{Decamter-Wave Measurements of Total Radar Cross Section}

Initial results of some early measurements at centimeter and meter wavelengths [Evans, 1962; Evans and Pettengill, 1963a, b] have suggested that the Moon displays a generally increasing radar cross section with increasing wavelength. A later measurement at $8.6 \mathrm{~mm}$ [Lynn, Sohigian, and Crocker, 1963, 1964], plus additional data in the centimeter and meter regions, has given rise to a modification of this view [Evans and Pettengill, 1963c]. At present, the earlier NRL measurements at decameter wavelengths are the only known evidence in favor of any discernible wavelength dependence in the Moon's total radar cross section. It should be mentioned, however, that little reliable work has been done until recently at wavelengths longer than $3 \mathrm{~m}$, except for the NRL 11- to $22-\mathrm{m}$ measurements, and the uncertainty in most shorter wavelength measurements is adequate at least to allow such a wavelength dependence to exist. An early set of measurements at 7.84 m [Evans and Ingalls, 1962] showed no apparent wavelength dependence in either the total radar cross section of the moon or in its behavior as a scatterer in depth. However, these measurements were relatively insensitive to energy reflected from regions beyond the Moon's central, specularly reflecting region, and their estimated error was quite large as well.

It is of utmost importance in experiments involving transionospheric radio propagation that contaminating effects of the earth's ionosphere be recognized and compensated. In lunar radar measurements these effects are superimposed upon other, more predictable modulations due to lunar libration and the antenna's radiation pattern. They combine in a particularly unfortunate manner at decameter wavelengths. Because most HF radio antennas are linearly polarized, decameter-wave lunar echoes are subject to deep fading due to the ionospheric Faraday effect, which imposes upon them a periodicity of from less than a minute to several minutes, as ionospheric undulations and continuous variation in the direction of the lunar ray vector bring about variations in total length of the phase path. Lunar libration fading, with a period varying between $2 \mathrm{sec}$ and $20 \mathrm{sec}$ at decameter wavelengths, contributes substantially to the short-period scintillation of the lunar echo as well.

On a longer time scale, traveling ionospheric irregularities often display an effective periodicity between 20 and $40 \mathrm{~min}$, causing potentially confusing focusing and defocusing of the Moon signal [Tveten, 1961; Tveten, Melanson, and Moller, 1965]. The effects of ionospheric irregularities are complicated in the case of the NRL HF facility in that the passage of the Moon through the antenna lobe structure occurs with this same approximate periodicity.

The consequence of these phenomena is that great caution must be exercised that only data is selected for analysis in which the four effects may be separated. It has been characteristic of the decameter-wave measurements conducted by NRL that many observations simply have had to be discarded in pursuit of this goal. The data presented below are examples of several measurements which have been obtained in which it is believed that these phenomena are adequately separable.

Figure 1 contains a representative sampling of total radar cross-section measurements which have been made between wavelengths of $8.6 \mathrm{~mm}$ and $19 \mathrm{~m}$. The earlier NRL measurements, at 11.3, 15.6, and $19.2 \mathrm{~m}$, which fall within the 0.1 to 0.2 level on figure 1, represent the evidence in favor of an increasing trend of total radar cross section with wavelength.

Recent improvements in the NRL HF radar facility have permitted a coherent integration (or predetection bandwidth narrowing) technique to be employed as

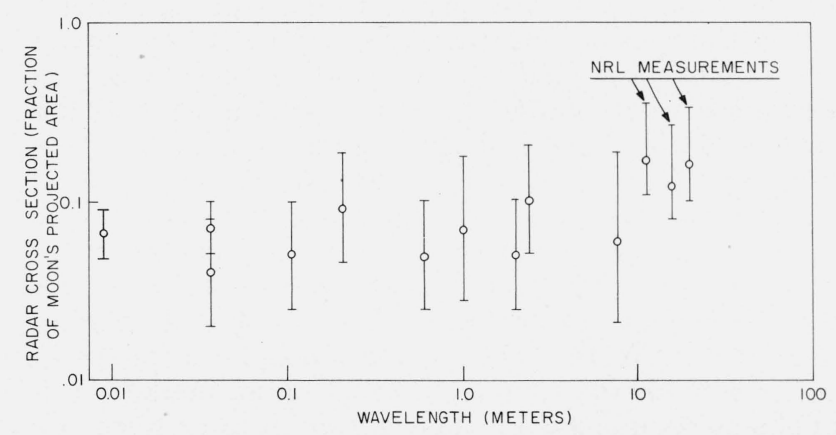

Figure 1. Radar cross section of lunar surface at wavelengths between $8.6 \mathrm{~mm}$ and $19.2 \mathrm{~m}$ [Evans and Pettengill, 1963b; Lynn, Sohigian, and Crocker, 1963|. 
an aid to eliminating the 2- to 20 -sec fading structure caused by lunar libration. Smoothing of echo information over a period of $20 \mathrm{sec}$ (of which $10 \mathrm{sec}$ represents the coherent integration period) has been utilized, and analysis of lunar echo data has been simplified and somewhat improved in accuracy relative to the means employed in determining the points in figure 1. Of the remaining variations, data have been selected for which fading due to the Faraday effect has been readily recognizable, and measurements have been restricted to 1-min periods at the peaks of the Faraday fading pattern. Data have been selected for which the antenna lobe structure could be easily discerned as well. The most difficult phenomenon to perceive, however, has been the recurrent focusing and defocusing effect of the earth's ionosphere, which has been the chief contaminant of lunar echo data. Its influence has been reduced by selection of modal values of total lunar radar cross section from a series of measurement periods. That is, several $1 \frac{1 / 2}{\mathrm{~h} h}$ observation periods have been analyzed, each of which has yielded 6 to $101-$ min mean values for the total radar cross section. Approximate modal values of these many mean cross-section measurements have been selected as the most accurate representation of the actual lunar radar cross section. The value of 0.17 times the Moon's projected area for the $11.3-\mathrm{m}$ radar cross section result in figure 1 has in large been confirmed by this improved analysis.

Figure 2 is an example of a typical series of radar cross-section measurements made at a wavelength of $11.3 \mathrm{~m}$ during the moonrise period of December 22, 1964. Total radar cross section is indicated along the left of each strip in figure 2, relative to the total projected area of the Moon $\left(A_{m}\right)$. Eastern Standard Time (which can be simply related to lunar elevation) appears along the abscissa. Antenna pattern nulls are apparent at 2125, 2150, and 2215 EST. Although rapid changes in amplitude are a common characteristic of the decameter-wave lunar echo, the sudden changes in signal amplitude at 2201:40 and 2221:30 EST are at least partially a result of antenna adjustments. It has been a necessary procedure at times to utilize the first few seconds of a Faraday rotation fading peak to optimize the azimuthal positioning of the antenna. The fading pattern exhibited by the remainder of figure 2 is an accurate indication of the true temporal behavior of

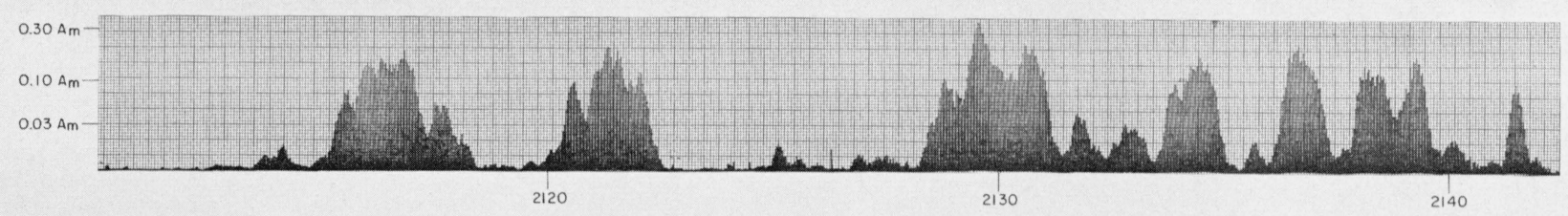

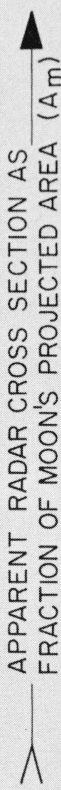
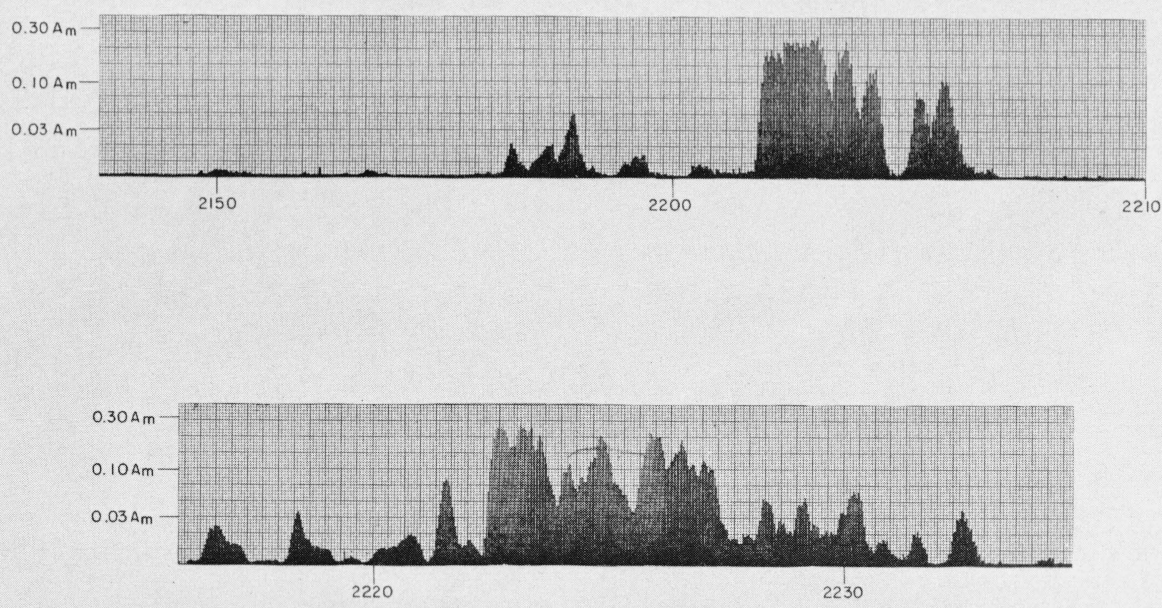

FiguRE 2. Radar cross section of the moon at $11.3 \mathrm{~m}$ measured on December 22, 1964. 
the lunar echo, subject only to the 20-sec smoothing introduced by the signal processor. In analyzing the data in figure 2, 10 polarization fading peaks, selected to occur near the center of each of three antenna lobes, were found to yield 1-min mean radar cross-section values grouped closely about $0.15 A_{m}$. It should be noted that there is little dispersion about this value; all except one of the 10 peaks fall within 10 percent of the mean.

A second characteristic of figure 2 which is deserving of mention is the depth of the Faraday rotation fading nulls. Those at 2119 (26 dB in depth), 2135:20 (24 $\mathrm{dB}$ in depth), 2137:30 (18 $\mathrm{dB}$ in depth), and 2204:30 (24 dB in depth) EST are of particular interest. All four occur near antenna-lobe peaks, and can reasonably be said to represent polarization fading exclu- sively. This circumstance emphasizes the extremely small contribution of depolarized energy to the received echo, and corroborates the view (discussed below) that the fraction of energy scattered from regions beyond the central specularly reflecting region is distinctly lower at decameter wavelengths than at shorter wavelengths. The data in figure 2 were sampled at a rate of one sample each 2 sec.

Figure 3 is a second example of radar cross-section measurements, made at $11.3 \mathrm{~m}$ on November 25 and 26, 1964, and displaying antenna nulls at 2340, 2400, 0050, and 0115 EST. Noisy conditions during the early portion of this observation led to the degraded character of the upper strip, and caused the data in the 2400 to 0055 EST time period to be discarded. The eight polarization fading peaks in figure 3 which

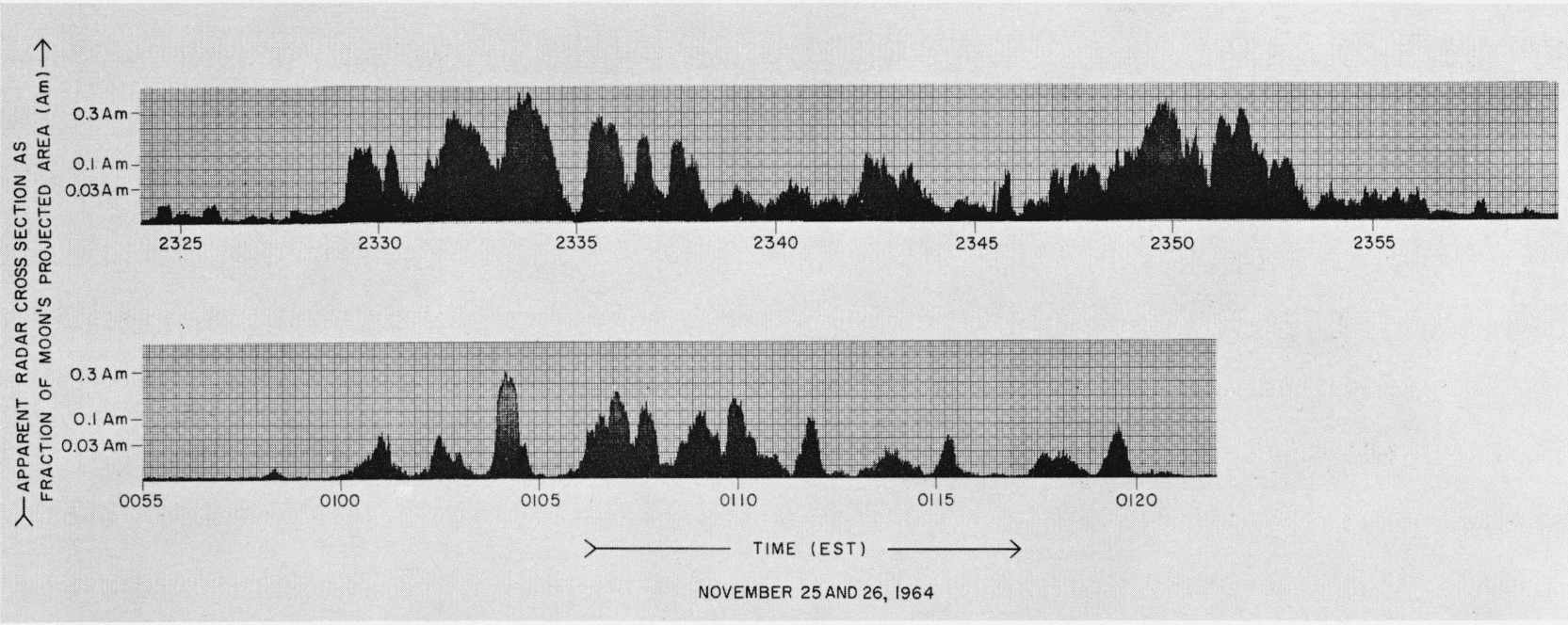

Figure 3. Radar cross section of the Moon at $11.3 \mathrm{~m}$ measured on November 25 and 26, 1964.

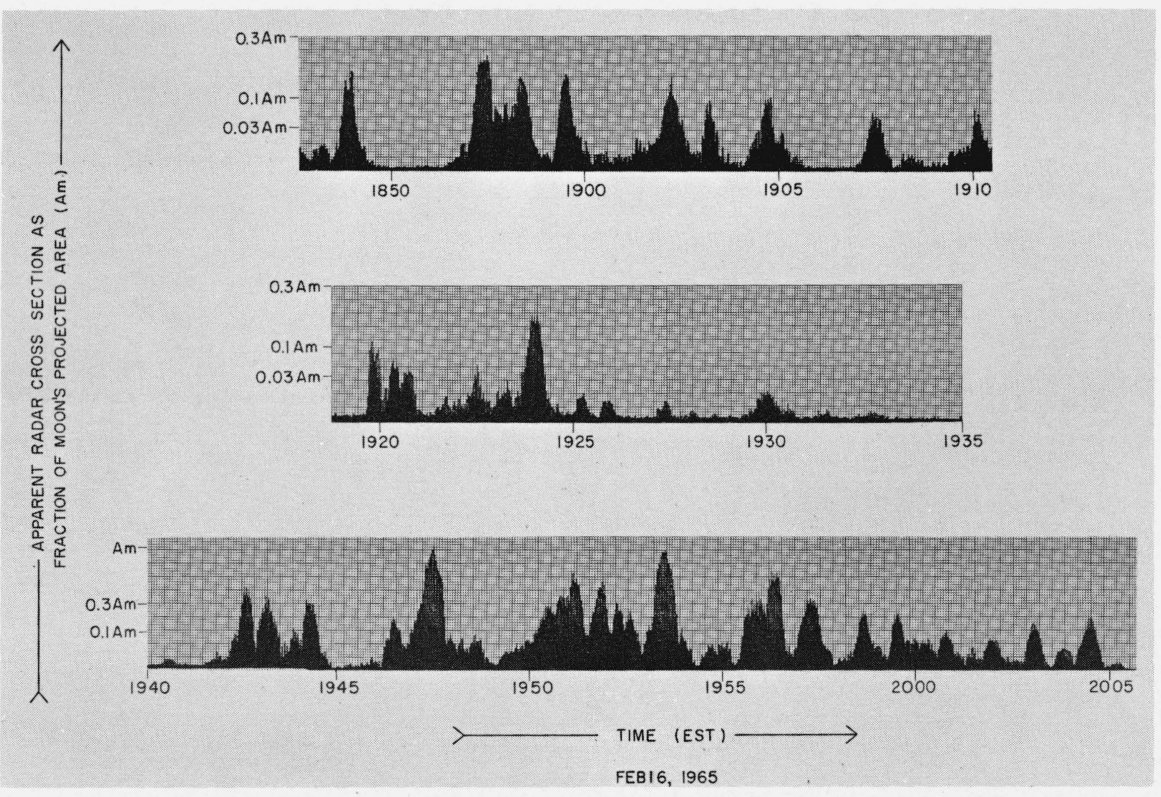

FIGURE 4. Radar cross section of the Moon at $11.3 \mathrm{~m}$ measured on February 16, 1965. 
coincide with antenna-lobe peaks give rise to 1-min mean cross-section values grouped about $0.18 A_{m}$. Polarization rotation fading nulls of $24 \mathrm{~dB}$ in depth are evident at 2335 and 0105 F.ST. The data in figure 3 were sampled at a rate of one sample per second.

Figure 4 contains measurements made during the moonrise period of February 16, 1965, also at $11.3 \mathrm{~m}$. These data were not scaled because of apparent amplitude anomalies, and are presented as an example of a supposed ionospheric focusing effect on the lunar echo. Antenna nulls occur in figure 4 at 1915, 1940, and 2005 EST. Notice that the bottom strip is plotted to a different scale from the two above it. Indeed, the amplitude of the two largest peaks on the bottom strip corresponds to an effective radar cross section nearly equal to the full projected area of the moon. It is only in the 1947 to 1957 EST time interval that such extremely large echoes appear; however, and it is presumed that their large magnitude is due to the passage of an ionospheric disturbance through the propagation path. It should be noted that the polarization fading pattern in figure 4 displays nulls of 20 to $27 \mathrm{~dB}$ in depth, in common with the other two examples.

\section{The Range Distribution of Energy Scattered by the Moon at Decameter Wavelengths}

Measurements at nearly all wavelengths between 8.6 $\mathrm{mm}$ and $11 \mathrm{~m}$ have suggested that the scattering distribution of radio energy by the Moon displays a distinct wavelength dependence. Whereas the lunar surface appears nearly uniformly bright at $8.6 \mathrm{~mm}$ [Lynn, 1963, 1964], it takes on an increasingly smoother appearance at longer wavelengths, until at $11 \mathrm{~m}$ the initial, quasi-specular component contains nearly all of the reflected energy. Evans' early measurements at $7.84 \mathrm{~m}$ did not indicate this trend [Evans and Ingalls, 1962], but his experimental apparatus was not capable of precise measurements of the low-amplitude echo components from regions beyond the central, specularly reflecting region.

Evidence regarding the scattering behavior of the moon may be gathered by three general methods:

(a) Actual measurements of echo power versus range may be made with a pulse radar, and an integrated composite pulse may be computed which represents an average scattering distribution over an extende 1 period. This is perhaps the most straightforward technique, but at decameter wavelengths care must be taken to avoid samples of data in which anomalous group path length changes occur due to ionospheric disturbances. Samples also must be chosen of adequate length such that apparently discrete scattering centers, or groups of temporarily in-phase scatterers, at ranges well beyond the Moon's leading edge, do not contribute a disproportionate fraction of the total received energy. The occurrence of these persistent echoes, at ranges up to $9 \mathrm{msec}$ beyond the initial quasi-specular component, has been a common phenomenon in the decameter-wave study conducted at NRL. (b) The spectral characteristics of the total Moon echo may be investigated, and an estimate of the scattering distribution may be extracted from this spectral data and known lunar libration parameters. This method is equivalent to, but somewhat less reliable at decameter wavelengths than, the ranging method because of continuous phase path variations in the ionosphere. It may be utilized in combination with the ranging method to perform a range-Doppler "mapping" of the lunar surface. A simple adaptation of this range-Doppler mapping technique has been made possible by modifications to the NRL HF signal processor.

(c) The contribution of the depolarized component in the total received echo may be measured as a rough indication of the fraction of the radar pulse which is reflected from effectively rough surfaces on the Moon. This contribution is expected to contain an appreciable portion of the total scattered energy from all regions of the Moon except the nearest, specularly reflecting region. Evidence of the extremely low depolarized component in the decameter-wave lunar echo is contained in the common 20- to $27-\mathrm{dB}$ null depth due to polarization fading in figures 2 to 4 .

The NRL-decameter-wave measurements have rested on the first of these techniques. Figure 5 is a comparison of the NRL results at $11.3 \mathrm{~m}$ with previous measurements by Evans [1962] and Lynn, Sohigian, and Crocker [1963]. It must be emphasized, however, that the $11.3-\mathrm{m}$ data in figure 5 have been taken from a limited number of 1- to 2-min samples, of 350 to 650 pulses each, and should be regarded as a best estimate from carefully chosen periods.

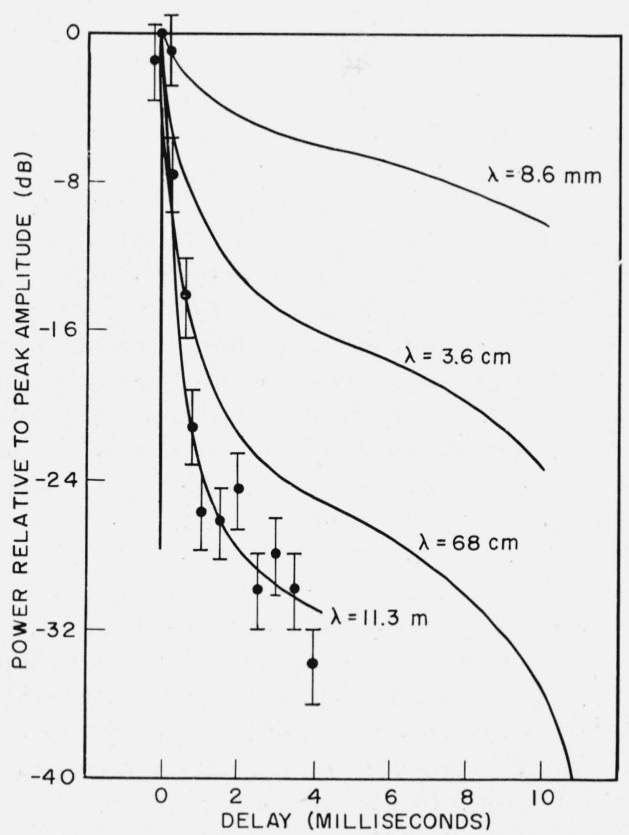

Figure 5. Composite Moon echoes measured at $8.6 \mathrm{~mm}, 3.6 \mathrm{~cm}$, $68 \mathrm{~cm}$, and $11.3 \mathrm{~m}$.

The 8.6-mm curve is from Lynn. Sohigian. and Crocker [1963]. and the 3.6- and 68-cm curves are from Evans |1962|. 


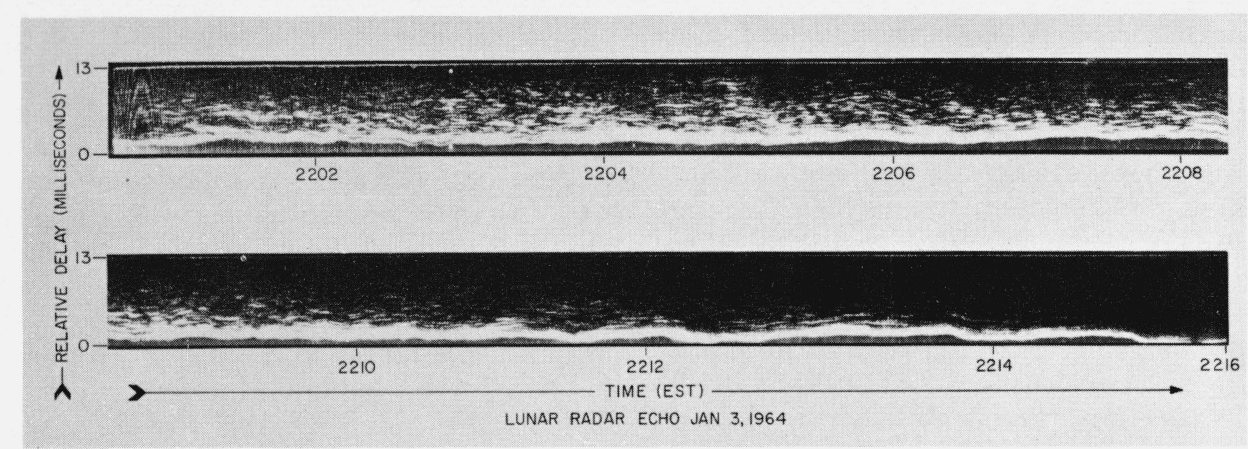

FiguRE 6. Range-time plot of 11.3-m lunar radar echo measured on January 3, 1964.

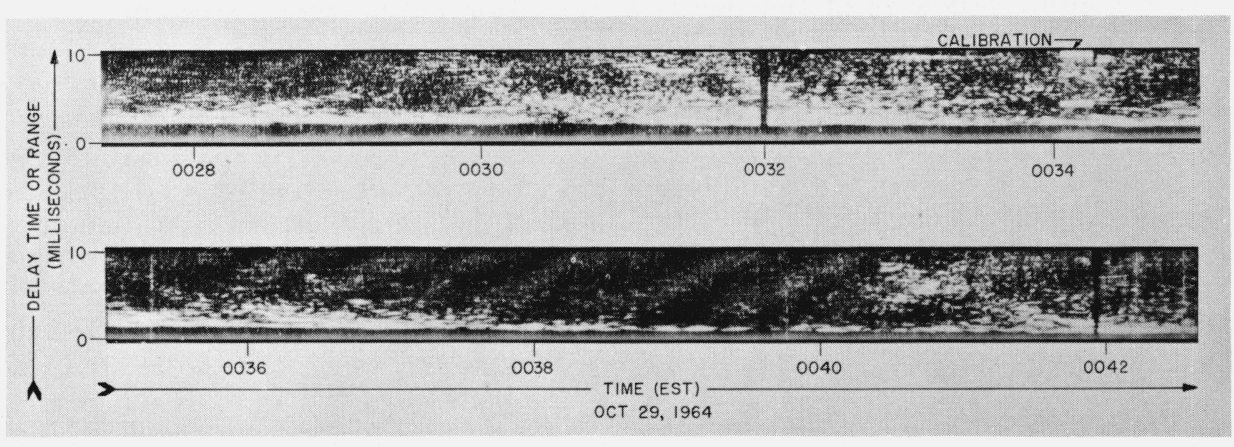

FiguRE 7. Range-time plot of 11.3-m lunar radar echo measured on November 29, 1964.

Ionospheric variations have precluded the extension of sampling periods beyond this 2-min limit, and data have been selected for which anomalous fading, focusing, or group-delay effects have been absent, and for which the integrity of the Moon pulse and a high signal-to-noise ratio have been preserved throughout the period. This procedure itself involves an unavoidable bias, and does not eliminate the possible effects of persistent, discrete echoes at points beyond the leading edge of the Moon pulse, which would be smoothed out if longer periods had been possible.

Figure 6 is a familiar $z$-plot, or range-versus-time plot, of the 11.3-m lunar echo received during a 15-min period of January 3, 1964, and is included here as an illustration of the anomalous echoes and group-delay effects which are commonly encountered at decameter wavelengths. The total range or delay time base in figure 6 is $13 \mathrm{msec}$, from bottom to top, and Eastern Standard Time is indicated from left to right. At the extreme top of the trace is a white fringe which corresponds to the start of the receiver blanking pulse associated with a subsequent transmit cycle. It has been allowed to appear on the photograph as assurance that the apparent group pathlength changes in figure 6 are not a result of synchronization instability. Fading due to polarization rotation is not seen in figure 6 because the Moon echo has been clipped at a low level to allow the low-amplitude components to be recorded. Notice the anomalous echo at 2201 to 2202 EST, which appears at the same time when the Moon, an approaching target in space, appears to recede briefly due to variations in group pathlength. Several short, 10- to
30 -sec duration, discrete echoes are evident in the 2203 to 2209 EST time interval. Notice in particular those at 2203 (6.5 msec delay beyond leading edge), 2204:40 (5.5 msec delay), and 2205 (9 msec delay). A second anomalous echo appears at 2208 . Notice also the group pathlength changes of over a millisecond in magnitude in the 2211 to 2216 time interval.

Figure 7 contains a second lunar echo $z$-plot, also at $11.3 \mathrm{~m}$ made on October 29, 1964. The time base in figure 7 is $10 \mathrm{msec}$ from bottom to top. There are no extensive group path changes in figure 7 , but strong, persistent, discrete echoes are evident at 0031:20 (6 msec delay beyond leading edge) and at 0033 (7.5 msec delay). Transmitter-off intervals at 0032 and at 0042 indicate the external noise level, and a calibration signal appears at 0034 . Notice also the wavelike character of the delayed echo component between 0030 and 0033 and between 0041 and 0042 . This phenomenon cannot easily be explained simply in terms of ionospheric modulations, and perhaps corresponds to libration-associated progression of phase fronts across the lunar surface. Some libration fading structure may be seen in the main component of the echo between 0038 and 0041 .

Consideration of phenomena such as those in figures 6 and 7 have convinced NRL decameter-wave experimenters that scattering distribution data at these wavelengths should be carefully selected and regarded with caution.

The existence of persistent, large-amplitude echoes at points well beyond the leading edge of the Moon pulse has suggested that a range-Doppler analysis 
technique might be of value in an attempt to resolve the question of their origin. That is, by measuring the relative Doppler shift and spectral distribution of these echoes, as well as their relative location in time delay, it should be possible to determine whether they may be associated with individual scattering centers, such as large craters which might have extensive planar surfaces associated with steeply sloping sides. The coherent integration technique which has been described in section 2 is performed with the aid of an effective $0.1-\mathrm{c} / \mathrm{s}$ filter, which may be combined with the $250-\mu$ sec range resolution afforded by the radar pulse length to yield a $250 \mu$ sec $\times 0.1 \mathrm{c} / \mathrm{s}$ resolution range-Doppler cell. This signal processor has only limited applicability to range-Doppler analysis in the decameter region, however, because the maximum libration-associated Doppler shift in this band never exceeds approximately $1 / 2 \mathrm{c} / \mathrm{s}$. Furthermore, the ionospheric phase-path modulations which have been found to have substantial effects upon decameterwave radio signals are expected to engender as much as $1 / 2 \mathrm{c} / \mathrm{s}$ additional dispersion in the lunar echo [Evans and Ingalls, 1962]. Once again, it appears, a cautious process of selection must be applied in choosing data for analysis.

Figure 8 contains a series of spectrum measurements, made with a $300 \mu \mathrm{sec} \times 2 \mathrm{c} / \mathrm{s}$ range-Doppler gate, which was applied to the lunar echo in figure 2 at 2130:25 EST. The gate was moved across the lunar echo in $250-\mu$ sec $(0.25-\mathrm{msec})$ steps, and meas- urements of the spectral distribution were made at each point from the beginning of the lunar pulse until it descended into noise. Amplitude is indicated on a linear scale along the ordinate, and Doppler shift appears on the abscissa. Notice that the first three photographs show a single, narrow component, whose $10-\mathrm{dB}$ bandwidth is approximately $0.2 \mathrm{c} / \mathrm{s}$. This bandwidth represents the resolution bandwidth of the analysis filter, and the signal in the first three photographs represents the large specular component of the echo. This component decreases rapidly in amplitude, and in the photograph at $1.5 \mathrm{msec}$ a clear split-Doppler echo is evident. It may be seen that the lower frequency component $(-0.12 \mathrm{c} / \mathrm{s})$ dominates until 2.50 msec, whereupon its spectrum broadens, possibly as a result of noise. A large, discrete echo appears at $-0.05 \mathrm{c} / \mathrm{s}$ in the photographs taken at 3.75- and $4.00-\mathrm{msec}$ delay. It is this type of echo which may be associated with a particular scattering center. Work has just begun on this study, however, and figure 8 is presented simply as an example of its capabilities. No attempt has been made as yet to associate these discrete range-Doppler echoes with individual regions on the lunar surface.

Figure 9 is a series of spectrum measurements made from data recorded approximately $1 / 2 \mathrm{~min}$ earlier. Once more, the initial specular component displays a narrow spectrum, which broadens as the amplitude decreases rapidly. A split-Doppler trace appears at 1.25 -msec delay, with components at $0.05 \mathrm{c} / \mathrm{s}$ on either

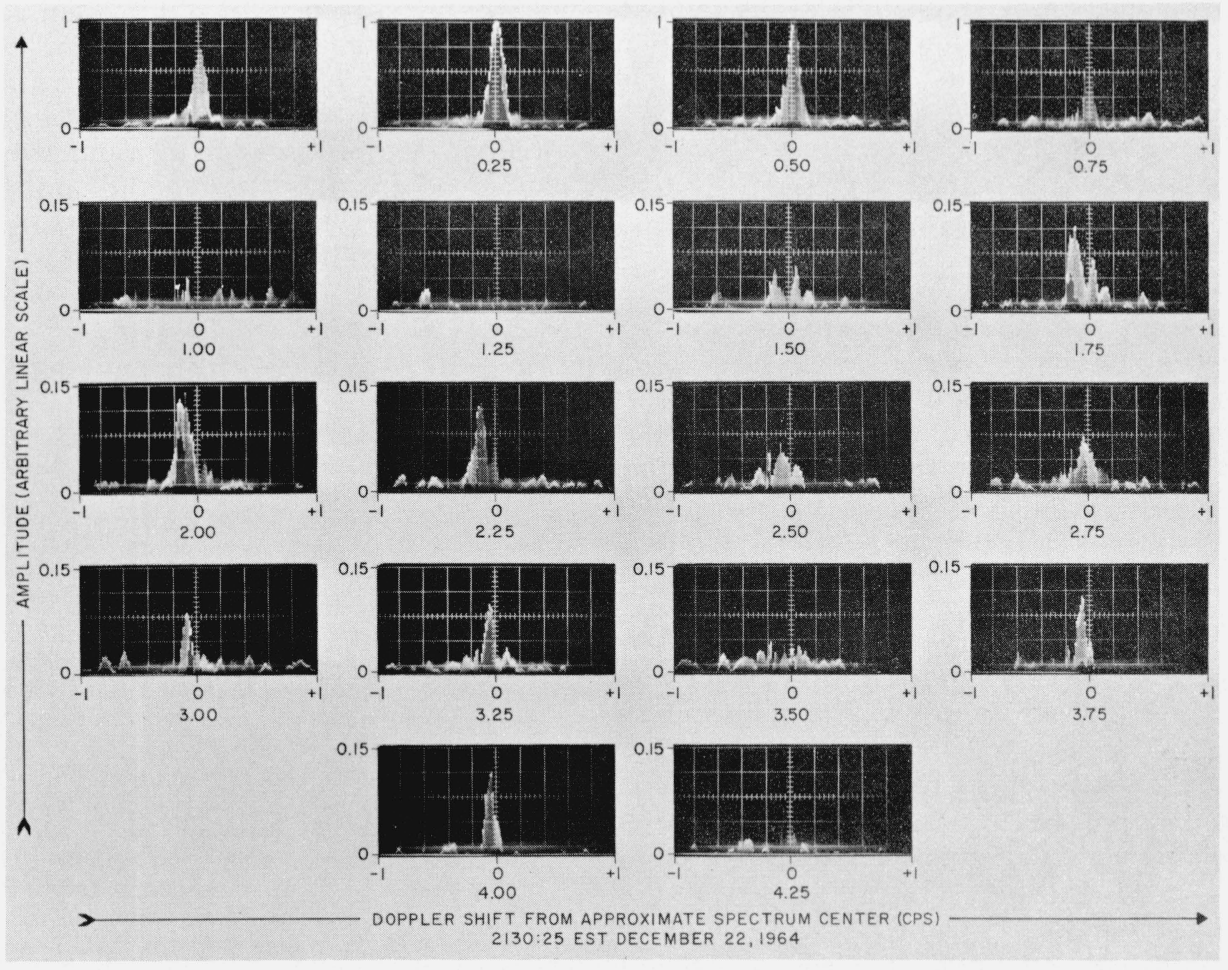

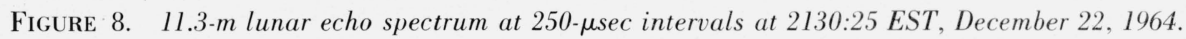




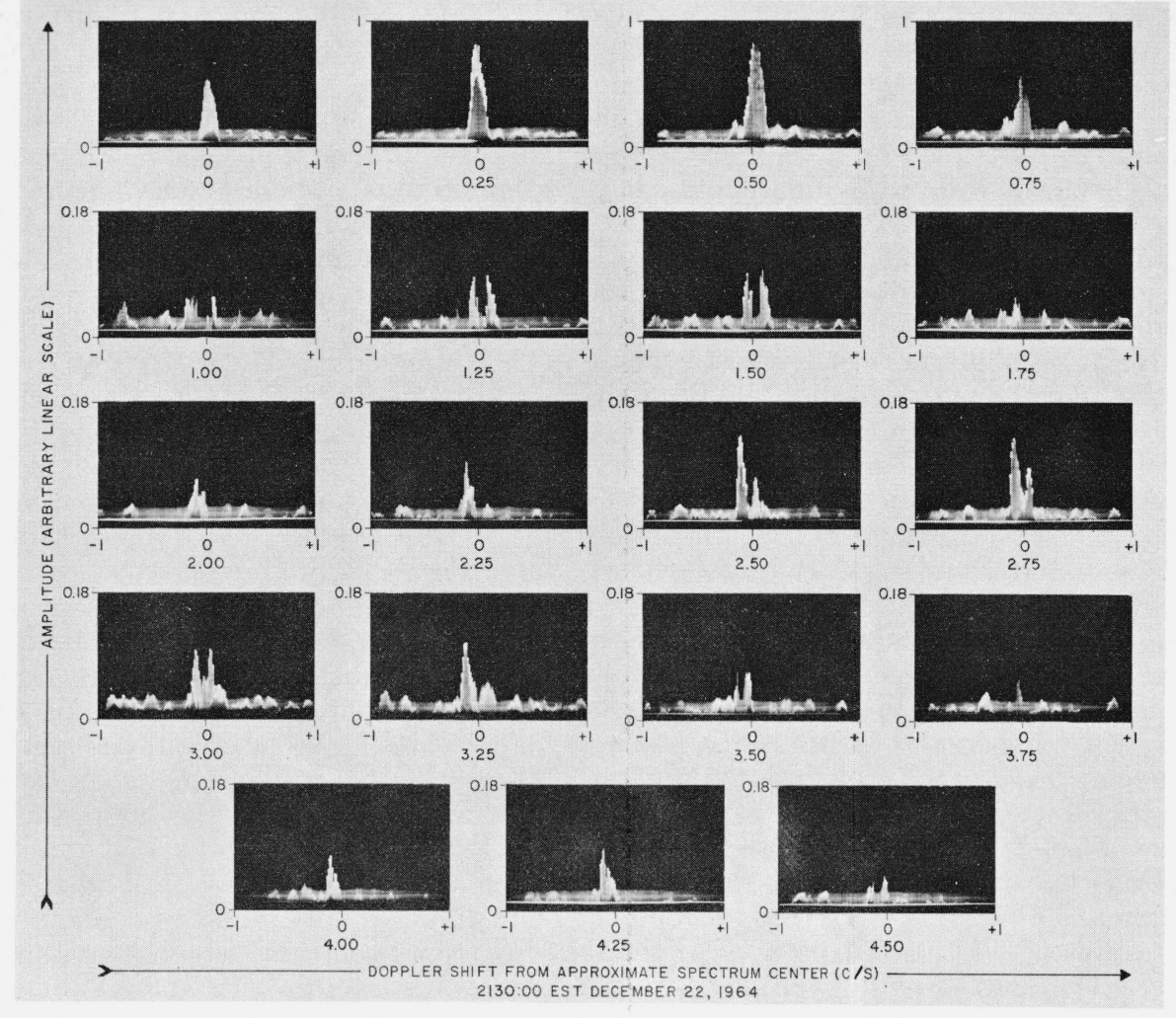

FIGURE 9. 11.3-m lunar echo spectrum at 250- $\mu$ sec intervals at 2130:00 EST, December 22, 1964.

side of the spectrum center. A similar echo appears at 2.00-msec delay with the lower component dominant. A large, discrete echo, at about $-0.1 \mathrm{c} / \mathrm{s}$, appears at 4.00 -msec delay.

\section{Conclusion}

It is believed that the radar cross section measurements presented in section 2 represent the limit of accuracy allowed by ionospheric processes in the decameter region. Recent, more precise measurements at $11.3 \mathrm{~m}$ corroborate the previous suggestion [Davis and Rohlfs, 1964] that the total lunar radar cross section is substantially larger in this region than at shorter wavelengths. A modal value of 0.17 times the Moon's projected area for this radar cross section value is indicated.

The strong effects of fluctuations in the ionosphere upon radio waves in the $\mathrm{HF}$ band require that all such measurements conducted over a transionospheric path be regarded with a note of caution, however, and that data be selected carefully to avoid contamination by ionospheric irregularities. Groupdelay anomalies involving changes of group pathlength in excess of 1 msec within a few minutes' duration are common. Anomalous echoes, which may be attributed to multipath phenomena, also are encountered frequently.
The occurrence of persistent, discrete, largeamplitude echoes from ranges well beyond the Moon's leading edge has led to an investigation of possible individual scattering centers toward the limb of the Moon. A simple form of range-Doppler analysis is employed for this study, and initial results indicate that echoes which are discrete in both range and Doppler can indeed be discerned.

\section{References}

Davis, J. R., and D. C. Rohlfs (Aug. 1, 1964), Lunar radio-reflection properties at decameter wavelengths, J. Geophys. Res. 69, No. $15,3257-3262$.

Evans, J. V. (June 22, 1962), Radio echo observations of the moon at 68-cm wavelength, Tech. Report No. 272, Lincoln Laboratory, M.I.T., Cambridge, Mass.

Evans, J. V., and R. P. Ingalls (Nov. 20, 1962), Radio echo studies of the moon at 7.84-meter wavelength, Tech. Report No. 288, Lincoln Laboratory, M.I.T., Cambridge, Mass.

Evans, J. V., and G. H. Pettengill (Jan. 15, 1963a), The scattering behavior of the moon at wavelengths of 3.6, 68 and 784 centimeters, J. Geophys. Res. 68, No. 2, 423-447.

Evans, J. V., and G. H. Pettengill (Sept. 1, 1963b), The Radar cross section of the moon. J. Geophys. Res. 68, No. 17, 5098-5099.

Evans, J. V., and G. H. Pettengill (1963c), The scattering properties of the lunar surface at radio wavelengths, The Moon, Meteorites, and Comets, ed. B. M. Middlehurst and G. P. Kuiper, pp. 129-161 (University of Chicago Press, Chicago).

Lynn, V. L., M. D. Sohigian, and E. A. Crocker (Oct. 8, 1963), Radar observations of the moon at 8.6-mm wavelength, Tech. Report No. 331, Lincoln Laboratory, M.I.T., Cambridge, Mass. 
Lynn, V. L., M. D. Sohigian, and E. A. Crocker (Feb. 15, 1964), Radar observations of the moon at a wavelength of 8.6 millimeters, J. Geo. Phys. Res. 69, No. 4, 781-783.

Tveten, L. H. (Mar--April, 1961), Ionospheric motions observed with high frequency backscatter sounders, J. Res. NBS 65D (Radio Prop.), No. 2, 115-127.

Tveten, L. H., L. L. Melanson, and H. G. Moller (March 1965), High Frequency Backscatter Studies, RADC Technical Report RADCTR $-65-39$.

\section{Discussion Following Davis et al.'s Paper}

I. Shapiro: In view of the extreme interest in accurate cross section measurements at long wavelengths, would it be possible to calibrate on a test sphere in orbit?

Answer: Yes, it would be useful to do so. We have looked at the Echo II satellite, but signals were too weak to be very helpful.
A. Giraud: Do you have trouble with meteor trails? Answer: Only in certain special situations and then we exclude the data.

T. Hagfors: Can you explain the variations in range of up to 2 msec?

Answer: We are currently exploring ray-tracing techniques.

G. H. Pettengill: Do you have a criterion for accepting or rejecting data which eliminates the possibility of personal bias?

Answer: Not with certainty.

T. Hagfors: How is the antenna gain measured?

Answer: Using measurements of scale models.

\title{
Lunar Mapping by Coherent-Pulse Analysis
}

\author{
T. W. Thompson \\ Cornell University
}

\author{
Arecibo Ionospheric Observatory, ${ }^{1}$ Arecibo, Puerto Rico
}

This paper describes a study of lunar radar echoes by a delay-frequency analysis which resulted in radar scattering maps of the moon. Figure 1 shows the moon as it was illuminated by the narrow antenna beam of the Arecibo Ionospheric Observatory radar. The echoes are sampled so the Doppler-shifted frequency spectrum of each delay was determined. Because of the finite length of the transmitter pulse length, the return at a particular delay must come from the range annulus $D$. Because the frequency processing admits a finite bandwidth, the return at a particular frequency must come from the frequency annulus $F$. Thus a return at a given range and frequency must come from the intersection of these two annuli, the points $P$ and $P^{\prime}$ of figure 1 . Since the

${ }^{1}$ Operated by Cornell University with the support of the Advanced Research Projects Agency under a research contract with the Air Force Office of Scientific Research, OAR.

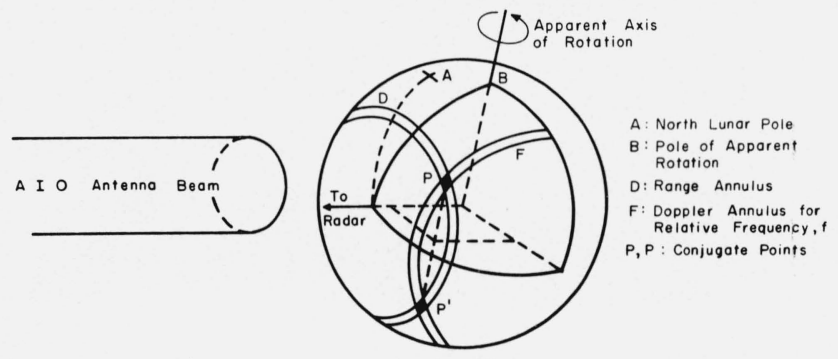

FIGURE 1. Geometry of the moon-radar system. area $P$ was within the antenna beam, the return must come from it and not from $P^{\prime}$.

In the radar scattering maps, the returns in range and frequency were mapped onto standard moon maps. These returns were expected to vary in range and frequency because of certain geometric effects, gain variation across the antenna beam, and the expected decay of reflected power with delays. These predicted variations were eliminated from the maps by using, instead of measured reflected power, the quantity

$$
\text { constant } x\left(\frac{P}{P_{0}}\right)
$$

where $P$ is the measured power, $P_{0}$ is the expected power based on the position of the reflecting area in the antenna beam and the known reflected power behavior of the moon. For convenience, the constant was chosen to make the numbers on the maps be near 100. The radar scattering maps then show normalized power returns plotted over their respective reflecting areas.

The result of one such radar mappings is shown in figure 2. The background mesh shows the delays and frequencies at which the returns were recorded. The numbers represent the normalized power returns. In the lower right-hand corner, the reflecting area for a single return is seen to be a parallelogram centered on an intersection of the mesh. The distances noted just above this parallelogram are typical for the resolution of our measurements. 